\title{
Feature Parameter Design Using Cross-sectional SEM for Machine Learning-based Optimization in Plasma Etching
}

Takashi Dobashi $^{1}$, Hyakka Nakada $^{2}$, Yutaka Okuyama ${ }^{3}$ and Takeshi Ohmori ${ }^{3}$

${ }^{1}$ Hitachi High Technologies America, Portland, Oregon, United States, ${ }^{2}$ Hitachi, Ltd., Research \& Development Group, Tokyo, Tokyo, Japan, ${ }^{3}$ Hitachi, Ltd., Research \& Development Group, Kokubunji, Tokyo, Japan

The structure of semiconductor elements in logic and memory devices such as the fin field-effect transistor (FinFET) and 3D-NAND cell has been miniaturized to the nanoscale. In the manufacturing processes for these elements, nanoscale accuracy is required, which makes developing the processes extremely difficult, costly, and time-consuming. There is thus a strong demand for a rapid process-development method. As plasma etching is one of the key processes in device manufacturing, we developed a method to optimize the plasma etching process by means of machine learning (ML) [1]. A plasma etcher needs a recipe with more than ten parameters for controlling nanoscale structures. In this study, we present a recipe optimization to obtain the deep vertical profile of an ultra-fine etching pattern with a line-and-space (L/S) mask made by self-aligned quadruple patterning (SAQP). Etching results are usually evaluated by feature values such as critical dimension (CD) and depth of the profiles in cross-sectional images observed by a scanning electron microscope (SEM) or transmission electron microscope (TEM). Our ML-based optimization method learns the relationship between recipes and profiles, so it is quite important to extract feature values that can evaluate the difference among the etching profiles corresponding to each recipe.

An in-lens type SEM was used for observing the cross-sectional images. A SEM specimen was prepared by cutting an etched sample in a direction perpendicular to the SAQP L/S pattern. A schematic of the initial profile is shown in Fig. 1(a). The width of an L/S was $12.5 \mathrm{~nm}$. Figure 1(b1) and (c1) show schematics of the vertical and bowing profiles, respectively, as typical etching results. Three feature parameters for evaluating the etching profiles were set as objective variables: depth, max CD, and bottom $\mathrm{CD}$ (Fig. 1(b1) and (c1)). Suppressing the bowing is key when it comes to achieving deep vertical etching. The bottom CD and max CD were selected as feature parameters because the degree of bowing can be evaluated from the difference in the length of both CDs. Depth was selected to evaluate the deepness of the trenches. Cross-sectional SEM images corresponding to Fig. 1(b1) and (c1) are shown in Fig. 1(b2) and (c2), respectively. The SAQP profile had periodic variation every four trenches stemming from the patterning process. Thus, the above parameters were measured past four trenches, and the total number of feature values was at least twelve.

We used an electron cyclotron resonance (ECR) plasma etcher for etching, as shown in Fig. 1(d). Process gases were introduced from the shower plate set on top of the reactor, and $2.45-\mathrm{GHz}$ microwaves were propagated into the reactor. ECR plasma was produced in the gases by the resonance between microwaves from the oscillator and the magnetic field by the dc-current of the coils. A recipe consisted of 11 explanatory variables with or without a breakthrough etching step and flow rates of ten gasses (e.g., CFbased ones and O2). A sample chip with SAQP on its surface was set on the wafer.

The relationship between recipes and etching profiles is nonlinear, and both have multiple variables. We used kernel ridge regression (KRR) to learn the relationship, as KRR is appropriate for nonlinear analysis with high-dimensional data since it takes advantage of the kernel method [2]. To construct a learning data set consisting of recipes and profiles, Si trench samples with SAQP were etched using 22 basic recipe conditions. By learning the data set using KRR, a model was obtained to predict the etching profiles of 
the Si trenches. Optimized recipes for obtaining vertical etching profiles were predicted by the model and verified in actual etching experiments. In the presentation, we will explain the details of the feature parameter design in cross-sectional SEM images for applying our ML-based method and show optimized etching results of SAQP samples.

(a)

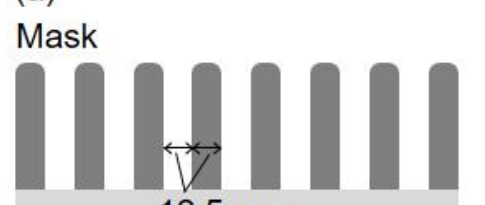

$12.5 \mathrm{~nm}$

\section{Silicon}

(d)

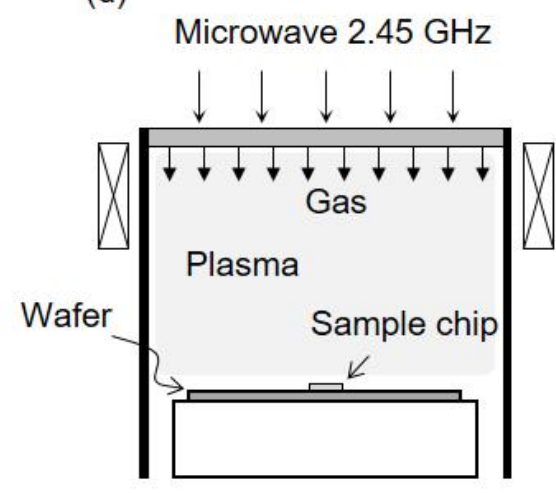

(b1)

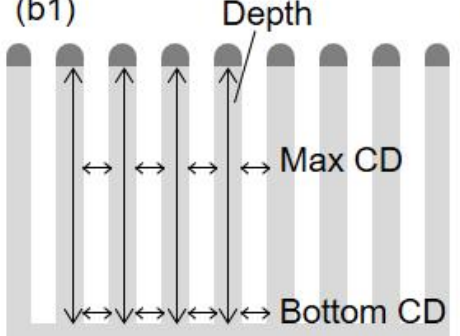

\section{(b2)}

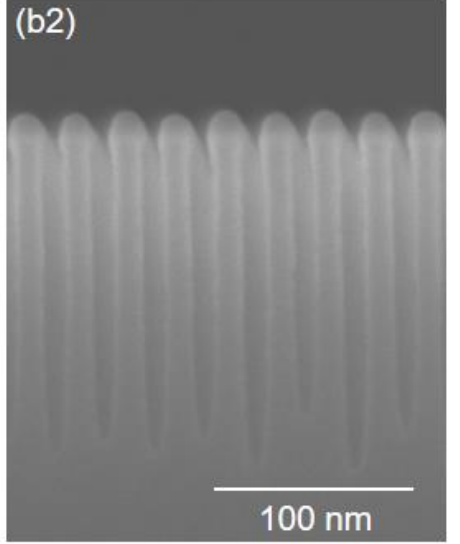

(c1)

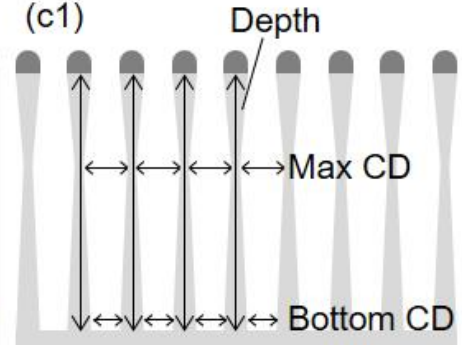

\section{(c2)}

Figure 1. Schematics of (a) initial sample with SAQP mask pattern and etching pattern of (b1) vertical and (c1) bowing profile. SEM image of (b2) vertical and (c2) bowing profile after etching. (d) Schematic diagram of ECR etcher.

\section{References}

1. T. Ohmori, et al., in Proc. Int. Symp. Dry Process, pp. 9-10, 2017.

2. J. Shawe-Taylor, et al., Kernel Methods for Pattern Analysis, Cambridge University Press, 2004. 